Monatsschr Kinderheilkd 2011 · 159:616-617 DOI 10.1007/s00112-010-2323-0

Online publiziert: 25. Mai 2011

(c) Springer-Verlag 2011
R. Kerbl $\mathbf{l}^{1} \cdot$ W. Sperl ${ }^{2}$

${ }^{1}$ Abteilung für Kinder und Jugendliche, Landeskrankenhaus Leoben

${ }^{2}$ Universitätsklinik für Kinder- und Jugendheilkunde Salzburg

\title{
Rehabilitation im Kindes- und Jugendalter
}

Das vorliegende Themenheft der Monatsschrift Kinderheilkunde beschäftigt sich mit Kinder- und Jugendlichenrehabilitation und versucht dabei, einen Überblick bzw. Einblick in renommierte Rehabilitationseinrichtungen und deren Arbeitsweise zu geben.

Glaubt man zu wissen, was mit Kinderrehabilitation gemeint ist, kommt man bei näherer Betrachtung zu der Erkenntnis, dass selbst innerhalb eines Landes Verständnis und Definition dieses Begriffs uneinheitlich sind. So dienen in Deutschland rehabilitative Maßnahmen bei Kindern und Jugendlichen nach Definition der Krankenkassen dazu,

„Gesundheitsstörungen oder deren Verschlimmerung zu verhüten",

nach Definition der Rentenversicherung jedoch auch dazu,

„den Patienten und seine Angehörigen in die Lage zu versetzen, möglichst unbeschränkt am sozialen Leben teilzuhaben".

In Österreich ist die Rechtslage noch wesentlich komplizierter und derzeit unbefriedigend, Kinder haben dort in den meisten Fällen momentan keinen rechtlichen Anspruch auf Rehabilitation.

Dabei darf es wohl als unbestritten gelten, dass sich Rehabilitationsmaßnahmen - ob mit dem Ziel der Verbesserung oder der Vermeidung von Verschlechterung bei Kindern und Jugendlichen aufgrund deren meist noch sehr langen Lebenszeit besonders rechnen und daher auch volkswirtschaftlich gut vertretbar sind. Dabei sind jedoch einige Grundregeln einzuhalten. Um eine Rehabilitation rechtfertigen zu können, muss eine entsprechende Rehabilitationsbedürftigkeit bestehen, die angeboren oder erworben sein kann. Des Weiteren müssen Rehabilitationsfähigkeit und -motivation vorliegen, d. h. die Rehabilitationsmaßnahmen müssen vom Patienten und dessen Eltern gewollt sein und ein bestimmtes Ziel verfolgen. Letzteres geht in die Rehabilitationsprognose mit ein. Im Idealfall lautet das Rehabilitationsziel

„vollständige Wiederherstellung/Erhaltung der Gesundheit und normale Teilhabe am sozialen Leben".

Es kann aber auch wesentlich niedriger angesetzt sein. Schlussendlich sollten nicht einmal Patienten mit infauster Prognose von rehabilitativen Maßnahmen ausgeschlossen bleiben, wenn durch diese die Verschlechterung der Erkrankung verzögert oder die Teilhabe am sozialen Leben zumindest vorübergehend verbessert werden können.

In den 4 Manuskripten dieses Themenheftes kommt der unterschiedliche Zugang zum Thema Kinderrehabilitation v. a. zwischen Deutschland und Österreich sehr klar zum Ausdruck. Während Deutschland über ein sehr dichtes Netz von Kinderrehabilitationskliniken verfügt, hat Österreich bisher kein einziges Kinderrehabilitationszentrum und muss bezüglich der Kinderrehabilitation als Entwicklungsland bezeichnet werden.

In der Arbeit von Sperl et al. wird dargestellt, welche Bemühungen zur Etablierung kinder- und jugendgerechter Rehabilitation derzeit in Österreich laufen. Dabei werden der Vorgang der Bedarfsermittlung erläutert und die verschiedenen aktuellen Indikationsbereiche beschrieben. Die Tatsache dass Österreich bezüglich Kinderrehabilitation ein Nach- zügler ist, mag auch den Vorteil haben, dass aus den Fehlern anderer u. U. gelernt werden kann. Im Blick über die Grenzen wird dies auch verdeutlicht: Während in Deutschland die Beschickung der Rehabilitationseinrichtungen zumindest $\mathrm{z}$. T. auch angebotsinduziert erscheint („A built bed is a filled bed"), wurden in Österreich in den letzten Jahren mit 2 verschiedenen Methoden eine Bedarfserhebung durchgeführt und ein Vorschlag für 3 über Österreich verteilte Rehabilitationszentren für Kinder und Jugendliche sowie entsprechende Qualitätskriterien erarbeitet. Im Mittelpunkt der Versorgung muss "das Kind/der Jugendliche" stehen und nicht (durch Integration in Erwachseneneinrichtungen) die „Gangstörung von o bis 100 “. Die Umsetzung des österreichischen Kinder- und Jugendlichenrehabilitationsplans bedarf allerdings noch des guten Willens zahlreicher Institutionen, darunter Gesundheitsministerium, Krankenversicherungen und Bundesländer, und vermutlich sogar einer Änderung des Allgemeinen Sozialversicherungsgesetzes (ASVG).

\section{( Die (Wieder-)Herstellung des bestmöglichen Zustands ist eines der Grundrechte von Kindern}

Dass Deutschland bezüglich Kinderrehabilitation über ein sehr gutes Angebot verfügt, wird in den 3 übrigen Manuskripten ersichtlich, die von Autoren aus bekannten Rehabilitationseinrichtungen verfasst wurden.

Zsoter et al. von der Rehabilitationsklinik Vogtareuth beschreiben in ihrem Beitrag die neurologische Frührehabili- 
tation bei Kindern und Jugendlichen. Als Indikationen nennen sie u. a. SchädelHirn-Traumen, hypoxische Hirnschädigungen, entzündliche Erkrankungen des Zentralnervensystems (ZNS), GuillainBarré-Syndrom, Schlaganfälle und Hirntumoren. Sie verweisen in ihrer Arbeit auf die Bedeutung der interdisziplinären Teamarbeit und die Notwendigkeit, in regelmäßigen Teamvisiten den Rehabilitationsfortschritt zu objektivieren und weitere Therapieziele festzulegen. Bei der Beurteilung des Rehabilitationsverlaufs berichten die Autoren über gute Erfahrungen mit einem Evaluierungstool, welches in seiner Kurzform als Remi-Pro (für Remissionsprofil) bezeichnet und an einem Fallbeispiel demonstriert wird. Sie verweisen auch auf die Notwendigkeit einer frühen Verlegung und einer guten Absprache mit dem Rehabilitationszentrum.

Bauer et al. vom bayrischen Rehabilitationszentrum Gaißach beschreiben die Rehabilitation des Asthma bronchiale im Kindes- und Jugendalter. Sie verweisen darauf, dass Asthma zu den häufigsten chronischen Erkrankungen zählt und aktuell in Deutschland etwa 600.00o Kinder und Jugendliche unter 17 Jahren betroffen sind. Eine (stationär erfolgende) Rehabilitation sehen sie für angezeigt, wenn trotz adäquater ärztlicher Betreuung beeinträchtigende körperliche, soziale oder psychische Krankheitsfolgen bestehen. Allein über die Rentenversicherungen werden jährlich etwa 8000 Kinder und Jugendliche (zum großen Teil unter 9 Jahren) mit dieser Indikation zugewiesen. Die Rehabilitation selbst stützt sich auf die Säulen Pharmako-, Klima-, Physio- und Sporttherapie, Patientenschulung und soziale Krankheitsbewältigung. Die Autoren betonen abschließend die Bedeutung definierter Standards und evaluierender QMVerfahren (QM: Qualitätsmanagement).

Schließlich berichtet $R$. Dopfer von der Nachsorgeklinik Tannheim über familienorientierte hämatoonkologische Rehabilitation. Er erwähnt, dass in Deutschland jährlich 1800 Kinder unter 15 Jahren an Krebs erkranken und dass für viele davon eine an die Akuttherapie anschließende Rehabilitation indiziert ist. Dabei gehe es nicht nur um die bestmögliche Wiederherstellung oder Erhaltung physischer Funktionen, sondern insbesondere auch um die Verbesserung der psychischen Befindlichkeit, die Stabilisierung des Familiensystems und die soziale Reintegration. Dies könnte in den meisten Fällen nur durch Einbeziehen der ganzen Familie gelingen, insbesondere auch der als Schattenkinder bezeichneten Patientengeschwister.

Wir hoffen, mit diesem Themenheft zur Rehabilitation im Kindes- und Jugendalter ein Thema adäquat anzusprechen, das zwar nur eine Minderheit unserer Kinder und Jugendlichen betrifft, aber gerade für jene besonders förderungsbedürftige Minderheit von großer Bedeutung ist.

Die (Wieder-)Herstellung des bestmöglichen physischen, psychischen und sozialen Zustands ist schließlich eines der Grundrechte von Kindern und Jugendlichen!

Leoben/Salzburg im Mai 2011

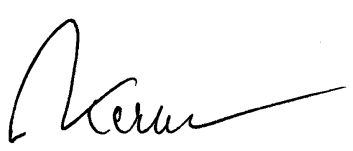

Prof. Dr. Reinhold Kerbl

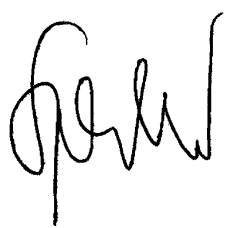

Prof. Dr. Wolfgang Sperl

\section{Korrespondenzadresse}

\section{Prof. Dr. R. Kerbl}

Abteilung für Kinder und

Jugendliche, Landeskrankenhaus Leoben Vordernbergerstraße 42, A-8700 Leoben

Österreich

reinhold.kerbl@lkh-leoben.at

\section{Impfungen: Fortschritte und Evidenz}

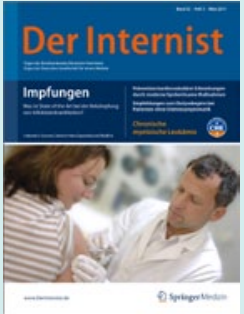

Impfungen gehören zu den fundamentalen Fortschritten der Medizingeschichte. Auch wenn kritische Töne nicht fehlen sollten, sind bestimmte Impfungen

für Erwachsene gerade im Zusammenhang mit der zunehmenden Reisetätigkeit essenziell. Für hochkontagiöse Infektionskrankheiten wie Masern oder Pertussis sind hohe Impfraten unverzichtbar, um einen Populationsschutz zu gewährleisten. Angesichts der Impfmüdigkeit innerhalb der Bevölkerung wird es deshalb immer wichtiger, aktiv über Impfungen zu informieren. Eine Zusammenstellung aktueller Impfempfehlungen bietet Der Internist in der Ausgabe 3/2011.

Die Schwerpunkte des Leitthemenheftes sind:

- Impfungen bei Erwachsenen

- Prävention der Virushepatitis A bis E

- Impfungen gegen Pneumokokken und Influenza. Wie groß ist die Evidenz?

- Impfungen unter immunsuppressiver Therapie chronisch entzündlicher Erkrankungen

Bestellen Sie diese Ausgabe zum Preis von $34,-€$ bei Springer Customer Service Center Kundenservice Zeitschriften

Haberstr. 7

69126 Heidelberg

Tel.: +49 6221-345-4303

Fax: +49 6221-345-4229

E-Mail: leserservice@springer.com

P.S. Vieles mehr rund um Ihr Fachgebiet finden Sie auf www.springermedizin.de 